\title{
Variações temporais na passagem de peixes pelo elevador da Usina Hidrelétrica de Santa Clara, rio Mucuri, leste brasileiro
}

\author{
Paulo dos S. Pompeu ${ }^{1} \&$ Carlos B. Martinez ${ }^{2}$ \\ ${ }^{1}$ Departamento de Biologia, Universidade Federal de Lavras. Campus Universitário, 37200-000 Lavras, Minas Gerais, Brasil. \\ E-mail: pompeu@ufla.br \\ ${ }^{2}$ Centro de Pesquisas Hidráulicas, Universidade Federal de Minas Gerais. Avenida Antônio Carlos 6627, 31270-901 Belo \\ Horizonte, Minas Gerais, Brasil.
}

\begin{abstract}
Temporal patterns of fish passage in Santa Clara Power Plant's fish lift, Mucuri River, east Brazil. The current study had the objective of determining temporal patterns of fish passage in Santa Clara Power Plant's fish lift, Mucuri River. During four months, from November 2003 to March 2004, transposition cycles were performed each two hours, when the specimens were counted and identified. During this migration period 67,838 individuals of 31 species passed through the lift. Migratory Characiformes abundance was larger during the day, whereas the Siluriformes were registered mainly at night. Fish abundance in the lift was significantly related to the Mucuri river flow. The temporal pattern found makes possible a better utilization of the lift, focusing cycles during the day and high flows periods.
\end{abstract}

KEY WORDS. Hydroelectric plants; passage; migration.

RESUMO. O presente estudo teve como objetivo determinar padrões temporais na passagem de peixes pelo elevador da Usina Hidrelétrica de Santa, localizado no rio Mucuri. Durante quatro meses, de novembro de 2003 a março de 2004, foram realizados ciclos de transposição a cada duas horas, quando todos os indivíduos foram contados e identificados. Durante este período foram transpostos 67.838 indivíduos de 31 espécies. A abundância de Characiformes migradores no elevador foi maior no período diurno, enquanto Siluriformes foram observados em maior quantidade à noite. A abundância de peixes no elevador foi relacionada significativamente com as vazões no rio Mucuri. O padrão encontrado torna possível a melhor utilização do elevador, priorizando sua operação durante o dia e em períodos de maior vazão.

PALAVRAS-CHAVE. Migração; passagens; usinas hidrelétricas.

O barramento de rios causa profundas modificações no ambiente aquático e nas comunidades presentes (SAlE 1985). Entre os empreendimentos responsáveis por estes impactos, destacam-se as barragens destinadas à formação de reservatórios de usinas hidrelétricas. O novo ambiente, formado após o barramento, apresenta características muito diferentes do original e as comunidades distinguem-se significativamente daquelas que ocorriam nos trechos lóticos originais ou remanescentes. Assim, o resultado inevitável destes empreendimentos, em relação à fauna aquática, é a alteração na abundância e riqueza de espécies, com proliferação de algumas e redução ou mesmo eliminação de outras (AgostinHo 1994). Entre as comunidades aquáticas, destacam-se os impactos sobre os peixes, principalmente por serem elementos mais facilmente visualizáveis no ambiente, por apresentarem valor econômico (pesca) e por serem sensíveis às mudanças ocorridas em outros componentes do sistema aquático.
Para as espécies de piracema as barragens constituem, em sua maior parte, obstáculos intransponíveis, alterando, ou mesmo impedindo o recrutamento em suas populações. Embora apenas uma pequena fração de nossas espécies seja de piracema (Petrere Jr. 1985, Godinho \& Godinho 1994), peixes migradores (ou de piracema), devido ao seu maior tamanho e sua maior abundância (NoRTHCOTE 1978), são os mais importantes para as pescas profissional (Goulding 1979, BitTenCourT \& Cox-Fernandes 1990, Godinho 1993) e amadora.

Muitas alternativas para minimizar os efeitos adversos dos barramentos sobre a migração dos peixes têm sido propostas e implementadas. Entretanto, este é um processo complexo que exige estratégias integradas entre diversas áreas e profissionais, em especial, biólogos e engenheiros.

Entre as estratégias empregadas para atenuar os efeitos do bloqueio exercido por barramentos na migração dos peixes está a construção de mecanismos de transposição, estruturas

Revista Brasileira de Zoologia 23 (2): 340-349, junho 2006 
hidráulicas que têm como objetivo principal permitir a subida e/ou descida dos peixes. A Legislação recente de alguns estados (Pará 1995, São Paulo 1997, Minas Gerais 1997, Esí́rito Santo 1999) tem tornado obrigatória sua construção em barragens, incluídas as destinadas à formação de reservatórios de Usinas Hidrelétricas.

Mecanismos de transposição de peixes são, essencialmente, condutos de água, através ou por volta de um obstáculo, que dissipam a energia hidráulica de maneira a permitir que o peixe possa subi-lo sem excessivo estresse (Clay 1995). São considerados mecanismos de transposição de peixes as escadas, eclusas e elevadores.

Na América do Sul já existem mais de 50 escadas em operação, distribuídas pelas diversas bacias hidrográficas (CLAY 1995). Elevadores e eclusas são menos comuns (QuiRós 1988), podendo ser citados os elevadores de Yacyretá e Porto Primavera no rio Paraná, a eclusa de Salto Grande, no rio Uruguay (Oldani et al. 1998), e o elevador de Funil, no rio Grande.

Elevadores para peixes são sistemas mecânicos no qual o peixe é capturado em um tanque apropriadamente dimensionado e localizado na base da barragem, sendo o mesmo posteriormente elevado e aberto a montante, permitindo a saída dos peixes em direção ao reservatório. É possível encontrar ainda uma variante desse mecanismo, conhecida como elevador tipo caminhão tanque. Nesse sistema, o peixe após ser capturado é conduzido a um tanque de um caminhão apropriado que fará o deslocamento de jusante para montante da barragem (CLAY 1995).

Este trabalho tem como objetivo o estudo das variações temporais na entrada de peixes no elevador da Usina Hidrelétrica de Santa Clara, implantada no rio Mucuri.

\section{MATERIAL E MÉTODOS}

\section{Área de estudos}

O rio Mucuri faz parte do conjunto de bacias independentes que drenam a região leste do Brasil. Sua área total de drenagem é de $15.100 \mathrm{~km}^{2}$, sendo 94,7\% dentro do território mineiro (Cетес 1983). O regime hidrológico da bacia do rio Mucuri caracteriza-se por duas estações bem definidas, seca e chuvosa, com maiores vazões de novembro a abril. A calha central do rio Mucuri pode ser dividida de acordo com suas características fisiográficas em duas unidades ambientais: seu alto e médio cursos apresentam maior declividade, predomínio de corredeiras e fundo de pedra; seu baixo curso, por outro lado, caracteriza-se como um sistema de deposição, com baixa declividade, fundo predominantemente arenoso, com a presença de áreas de Mata Atlântica sazonalmente alagadas, utilizadas para a plantação de cacau, e áreas de mangues e brejais na sua porção distal. A região localizada junto à divisa dos estados de Minas Gerais e Bahia representa a transição entre estas duas unidades do rio Mucuri.

A usina Hidrelétrica de Santa Clara está localizada no rio Mucuri, no limite dos municípios de Mucuri, Bahia; Nanuque e Serra dos Aimorés, Minas Gerais. A região de implantação da barragem coincide com a região de transição entre o baixo e médio cursos do rio Mucuri, a cerca de $80 \mathrm{~km}$ da foz, onde se localizavam as corredeiras de Santa Clara (Fig. 1).

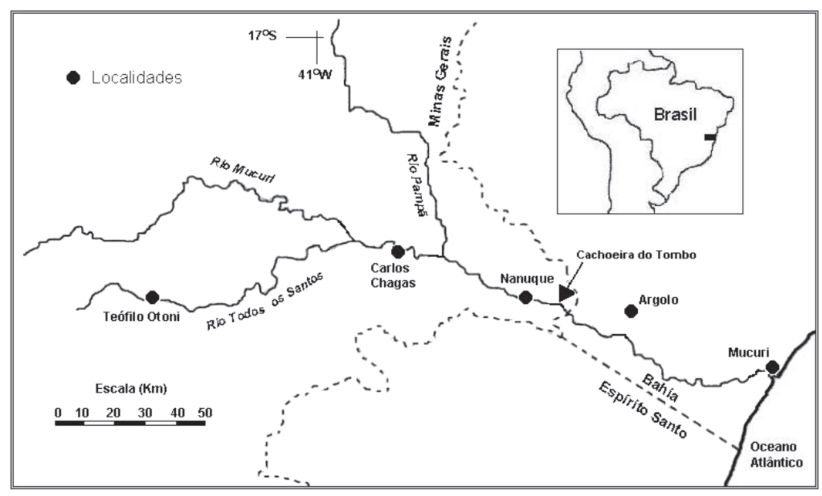

Figura 1. Mapa da bacia do Rio Mucuri, indicando as principais cidades da região e a localização da Usina Hidrelétrica de Santa Clara.

As obras para a construção da barragem iniciaram-se em 1999, sendo que a primeira turbina entrou em operação em fevereiro de 2002. Sua barragem de concreto possui $60 \mathrm{~m}$ de altura máxima sobre as fundações e $240 \mathrm{~m}$ de comprimento. Em novembro de 2003, entrou em operação junto à barragem da Usina Hidrelétrica Santa Clara um mecanismo de transposição de peixes do tipo elevador com caminhão-tanque. Este é composto por uma tubulação de água para atração, um canal de entrada e captura de peixes, um elevador e um caminhão-tanque.

A atração dos peixes é feita através de um conduto metálico ligando o reservatório ao canal do Sistema de Transposição. Este sistema de adução fornece um escoamento de água ininterrupto de até $3 \mathrm{~m}^{3} / \mathrm{s}$, propiciando a atração dos peixes existentes no Canal de Fuga da usina para o interior do canal do sistema de transposição. A cada duas horas, os peixes que se encontram no interior do canal são aprisionados e empurrados por um sistema de grades para a região da caçamba, com $4 \mathrm{~m}^{3}$ de capacidade, que se encontra submersa. A caçamba é içada e transportada até o caminhão-tanque. Com a caçamba posicionada sobre o tanque, os peixes são transferidos para o caminhão, também com capacidade de $4 \mathrm{~m}^{3}$, e transportados até o reservatório, onde são liberados.

\section{Métodos}

No dia 19 de novembro de 2003 teve início a operação do elevador com caminhão-tanque da Usina Hidrelétrica Santa Clara, que foi conduzida ao longo de quatro meses, até o dia 19 de março de 2004. Ao longo deste período, de segunda a sábado, foram realizados seis ciclos diários de transposição (atração, captura, transporte e liberação), com transporte nos horários de 8:00, 10:00, 12:00, 14:00, 16:00 e 18:00 h. Com freqüência quinzenal, também foram realizados ciclos de trans- 
posição noturnos, nos horários de 20:00, 22:00, 24:00, 02:00, 4:00 e 6:00 h. Durante todo o período a água de atração permanecia sendo liberada continuamente.

No total, foram realizados 636 ciclos de transposição, 588 diurnos e 48 noturnos, sendo que, em cada um deles, todos os indivíduos foram contados e identificados antes de sua liberação no reservatório. Para as espécies com dúvidas taxonômicas, exemplares eram fixados em solução de formol (10\%), para posterior identificação em laboratório.

Para cada espécie, foi calculada a captura por unidade de esforço diária (CPUE), para os períodos noturno e diurno, e para intervalos de duas horas ao longo do dia, segundo a equação: CPUE $=\mathrm{N}_{\mathrm{i}} / \mathrm{N}_{\mathrm{C}}$, onde: $\left(\mathrm{N}_{\mathrm{i}}\right)$ número de indivíduos transpostos da espécie $\mathrm{i} ;\left(\mathrm{N}_{\mathrm{c}}\right)$ número de ciclos de transposição no período avaliado.

Para estes cálculos foram excluídos os dados relativos às transposições realizadas no horário das 8:00 h, quando não eram efetuadas transposições noturnas. Adotou-se este procedimento porque nestas ocasiões as capturas representavam o resultado de 14 horas de atração, e não de duas horas. Para estas análises foi sempre considerado a hora real, independente do horário oficial de verão.

Dados diários de vazão no rio Mucuri e precipitação local foram obtidos junto à equipe de operação da Usina Hidrelétrica Santa Clara e no Posto Telemétrico de Nanuque, respectivamente. As relações entre o número de peixes transpostos e a precipitação diária em Nanuque e as vazões do rio Mucuri a jusante da barragem da Usina Hidrelétrica Santa Clara foram testadas através de análise de regressão simples, para cada variável ambiental, e através de regressão múltipla, para precipitação e vazão conjuntamente (LuDwig \& REYNOLDS 1988).

Como não existem estudos sobre a migração dos peixes no rio Mucuri, foram consideradas potencialmente migradoras espécies dos gêneros Leporinus, Prochilodus e Brycon, que em outras bacias sul americanas possuem representantes com comportamento migrador conhecido (LUCAS \& BARAS 2001).

\section{RESULTADOS}

Foram transpostos durante os quatro meses de operação do elevador, 67838 exemplares de 31 espécies de peixes, incluindo cinco de origem marinha: Centropomus undecimalis (Bloch, 1792), Centropomus parallelus Poey, 1860, Mugil curema Valencienes, 1836, Caranx latus Agassiz, 1829 e Achirus lineatus (Linnaeus, 1758). Neste período, picos de vazão no rio Mucuri e de precipitação na cidade de Nanuque foram observados, com maior intensidade, nos meses de janeiro e fevereiro (Fig. 2).

Os lambarís Astyanax bimaculatus (Linnaeus, 1758) e Astyanax intermedius Eigenmann, 1908 e as potenciais migradoras (Prochilodus vimboides Kner, 1859; Leporinus conirostris Steindachner, 1875; Leporinus steindachneri Eigenamnn, 1907 e Brycon ferox Steindachner, 1877) foram os mais abundantes, representando, juntos, 96\% dos indivíduos transpostos (Tab. I).

Em média, foram transpostos 106 peixes por ciclo, com

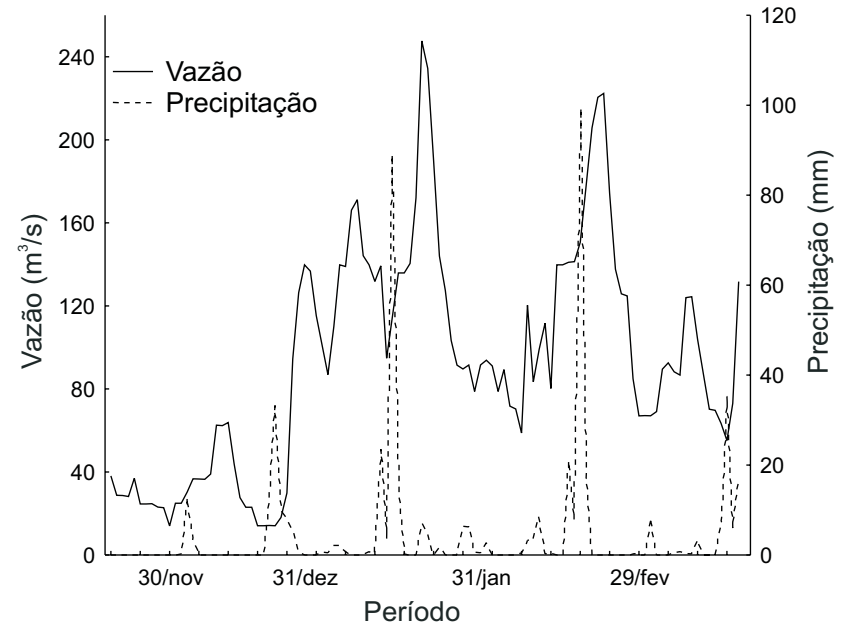

Figura 2. Precipitações diárias e vazões afluentes na região da barragem da UHE Santa Clara durante o período de estudos.

máximo de 5021, sendo que em 255 ciclos não foi transposto nenhum peixe (Tab. II). Nos ciclos com mais de mil indivíduos transpostos, grandes cardumes de lambaris ( $A$. bimaculatus e $A$. intermedius) ou de duas ou três espécies migradoras foram sempre responsáveis por mais de $90 \%$ das capturas (Tab. III).

Das espécies transpostas, 13 podem ser consideradas diurnas e quatro noturnas. Para as outras 14 espécies, não foi possível o estabelecimento do período preferencial de atividade devido ao pequeno número de indivíduos capturados (Tab. IV). No entanto, os dois períodos apresentaram capturas totais por unidade de esforço equivalentes, da ordem de 88 indivíduos por ciclo, considerando-se todo o período estudado.

Dos grandes grupos de peixes da região, Characiformes potencialmente migradores e peixes marinhos tiveram, na maioria dos casos, suas capturas concentradas no período diurno. Por outro lado, três das quatro espécies mais abundantes à noite foram de Siluriformes. Considerando-se todos os espécimens transpostos, podem ser individualizados dois grandes picos de captura pelo elevador: um entre 10:00 e 12:00 h e outro entre 18:00 e 22:00 h (Fig. 3).

Todas as espécies consideradas potencialmente migradoras apresentaram picos de abundância no elevador em torno do meio do dia. Das espécies que tiveram a distribuição das capturas ao longo do dia analisadas, apenas o lambari $(A$. bimaculatus) e os Siluriformes Trachelyopterus striatulus (Steindachner, 1877) e Pogonopoma wertheimeri (Steindachner, 1867) tiveram suas capturas por unidade de esforço maiores durante o período noturno (Fig. 4).

Para o conjunto de dados analisados, a vazão do rio Mucuri a jusante da barragem da Usina Hidrelétrica Santa Clara foi a principal variável explicativa da abundância de indivíduos transpostos (Tab. V). Para a ordem dos siluriformes, a precipitação também contribuiu significativamente com o modelo ajustado, sendo para este grupo, a principal variável explicativa. 
Tabela I. Lista das espécies de peixes transpostas pelo elevador para peixes da Usina Hidrelétrica Santa Clara, com o respectivo número total e abundância relativa dos indivíduos transpostos.

\begin{tabular}{llll} 
Espécies & Nome popular & \multicolumn{2}{c}{ Abundância } \\
\cline { 3 - 4 } &
\end{tabular}

\begin{tabular}{|c|c|c|c|}
\hline \multicolumn{4}{|l|}{ Characiformes } \\
\hline \multicolumn{4}{|l|}{ Characidae } \\
\hline Astyanax bimaculatus (Linnaeus, 1758) & Lambari-do-rabo-amarelo & 5.694 & 8,39 \\
\hline Astyanax intermedius Eigenmann, 1908 & Lambari & 24.775 & 36,52 \\
\hline Brycon ferox Steindachner, 1877 & Piabanha & 1.075 & 1,58 \\
\hline Brycon vermelha Lima \& Castro, 2000 & Vermelha & 2 & 0,00 \\
\hline Oligosarcus acutirostris Menezes, 1987 & Bocarra & 48 & 0,07 \\
\hline \multicolumn{4}{|l|}{ Erythrinidae } \\
\hline Hoplias malabaricus (Bloch, 1829) & Traíra & 24 & 0,04 \\
\hline \multicolumn{4}{|l|}{ Anostomidae } \\
\hline Leporinus conirostris Steindachner, 1875 & Piau-branco & 5.825 & 8,59 \\
\hline Leporinus copelandii Steindachner, 1875 & Piau-mutengo & 38 & 0,06 \\
\hline Leporinus steindachneri Eigenmann, 1907 & Piau-capim & 4.943 & 7,29 \\
\hline \multicolumn{4}{|l|}{ Curimatidae } \\
\hline Cyphocharax gilbert (Quoy \& Gaimard, 1824) & Sagüiru & 439 & 0,65 \\
\hline \multicolumn{4}{|l|}{ Prochilodontidae } \\
\hline Prochilodus costatus Valencienes, 1850 & Curimatá & 24 & 0,04 \\
\hline Prochilodus vimboides Kner, 1859 & Curimatá & 23.012 & 33,92 \\
\hline \multicolumn{4}{|l|}{ Gymnotiformes } \\
\hline \multicolumn{4}{|l|}{ Gymnotidae } \\
\hline Gymnotus carapo Linnaeus, 1758 & Sarapó & 1 & 0,00 \\
\hline \multicolumn{4}{|l|}{ Siluriformes } \\
\hline \multicolumn{4}{|l|}{ Auchenipteridae } \\
\hline Trachelyopterus striatulus (Steindachner, 1877) & Cumbaca & 71 & 0,10 \\
\hline Pseudauchenipterus affinis (Steindachner, 1877) & Ferrolho & 15 & 0,02 \\
\hline \multicolumn{4}{|l|}{ Pimelodidae } \\
\hline Pimelodella lateristriga (Lichtenstein, 1823) & Mandi & 4 & 0,01 \\
\hline \multicolumn{4}{|l|}{ Heptapteridae } \\
\hline Rhamdia quelen (Quoy \& Gaimard, 1824) & Bagre & 10 & 0,01 \\
\hline \multicolumn{4}{|l|}{ Trichomycteridae } \\
\hline Trichomycterus alternatus (Eigenmann, 1917) & Cambeva & 15 & 0,02 \\
\hline \multicolumn{4}{|l|}{ Loricariidae } \\
\hline Hypostomus affinis (Steindachner, 1877) & Cascudo-areia & 3 & 0,00 \\
\hline Hypostomus luetkeni (Steindachner, 1877) & Cascudo-chicote & 1 & 0,00 \\
\hline Pogonopoma wertheimeri (Steindachner, 1867) & Cascudo-preto & 670 & 0,99 \\
\hline \multicolumn{4}{|l|}{ Perciformes } \\
\hline \multicolumn{4}{|l|}{ Cichlidae } \\
\hline Cichla monoculus Spix \&Agassiz, 1831 & Tucunaré & 27 & 0,04 \\
\hline Geophagus brasiliensis (Quoy \& Gaimard, 1824) & Cará & 42 & 0,06 \\
\hline Oreochromis niloticus (Linnaeus, 1758) & Tilápia & 5 & 0,01 \\
\hline \multicolumn{4}{|l|}{ Gobiidae } \\
\hline Awaous tajasica (Lichtenstein, 1823) & Peixe-flor & 6 & 0,01 \\
\hline & & & Cont \\
\hline
\end{tabular}


Tabela I.Continuação.

\begin{tabular}{|c|c|c|c|}
\hline \multirow{2}{*}{ Espécies } & \multirow{2}{*}{ Nome popular } & \multicolumn{2}{|c|}{ Abundância } \\
\hline & & $\mathrm{N}$ & $\%$ \\
\hline \multicolumn{4}{|l|}{ Carangidae } \\
\hline Caranx latus Agassiz, 1831 & Xaréu & 1 & 0,00 \\
\hline \multicolumn{4}{|l|}{ Centropomidae } \\
\hline Centropomus paralellus Poey, 1860 & Robalo & 350 & 0,52 \\
\hline Centropomus undecimalis (Bloch, 1792) & Robalo & 541 & 0,80 \\
\hline \multicolumn{4}{|l|}{ Sciaenidae } \\
\hline Pachyurus adspersus Steindachner, 1879 & Corvina & 4 & 0,01 \\
\hline \multicolumn{4}{|l|}{ Mugilidae } \\
\hline Mugil curema Velenciennes, 1836 & Tainha & 172 & 0,25 \\
\hline \multicolumn{4}{|l|}{ Pleuronectiformes } \\
\hline \multicolumn{4}{|l|}{ Achiridae } \\
\hline Achirus lineatus (Linnaeus, 1758) & Linguado & 1 & 0,00 \\
\hline Total geral & & 67.838 & 100,00 \\
\hline
\end{tabular}

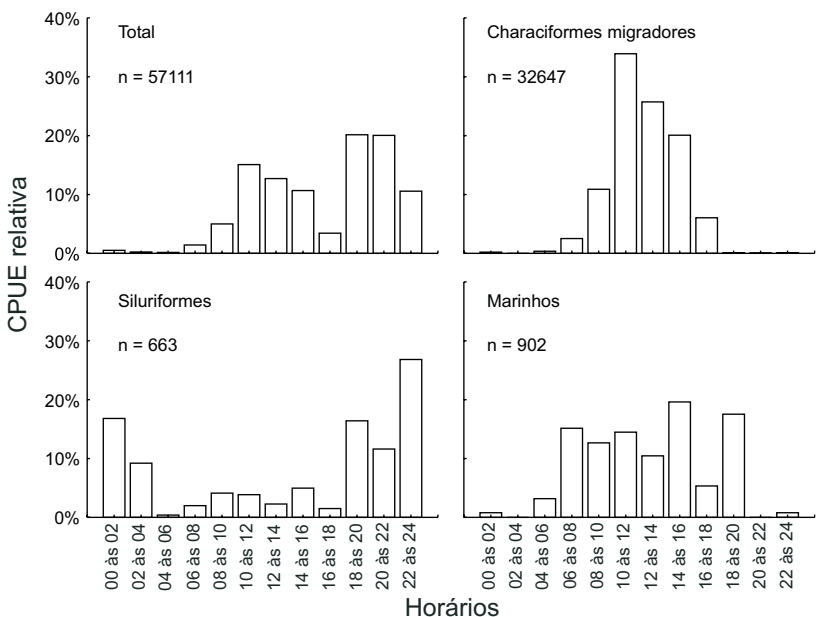

Figura 3. Distribuição das capturas totais, de Characiformes migradores, Siluriformes e peixes marinhos ao longo do dia, no elevador da Usina Hidrelétrica Santa Clara.

\section{DISCUSSÃO}

Em ambientes tropicais, é evidente a relação entre as variações no nível fluviométrico e os deslocamentos migratórios dos peixes, (Lowe-McConNell 1999), associados à maior atividade reprodutiva durante as cheias (Vazzoler 1996, Agostinho et al. 2000).

Estudos já haviam demonstrado os efeitos deste sincronismo sobre o fluxo de peixes em mecanismos de transposição Sul Americanos. Avaliando a ascensão de peixes na escada experimental de Itaipú, sob condições de velocidade e vazão constante na escada, constatou-se uma influência significativa da
Tabela II. Número absoluto e relativo de ciclos por classe de número de indivíduos transpostos.

\begin{tabular}{ccc}
\hline \multirow{2}{*}{ Indivíduos transpostos } & \multicolumn{2}{c}{ Ciclos de transposição } \\
\cline { 2 - 3 } & $\mathrm{N}$ & $\%$ \\
\hline $0 \leq \mathrm{N}<10$ & 339 & 0,53 \\
$10 \leq \mathrm{N}<100$ & 202 & 0,32 \\
$100 \leq \mathrm{N}<1000$ & 76 & 0,12 \\
$\mathrm{~N} \geq 1000$ & 19 & 0,03 \\
\hline
\end{tabular}

temperatura da água e da vazão turbinada pela Usina Hidrelétrica de Itaipu sobre a intensidade de ascensão dos peixes (FERnANDEZ et al. 2001). Na escada da Usina Hidrelétrica Igarapava, a maior parte dos peixes (75\%) utiliza o mecanismo de Outubro a Fevereiro, período correspondente à estação chuvosa na região (VONO et al. 2004). No elevador de Yacyretá, rio Paraná, espécies migradoras foram mais abundantes entre outubro e março (OlDANI \& BAIGÚN 2002).

Para as espécies dos gêneros Leporinus, Prochilodus e Brycon, a presença no elevador da Usina Hidrelétrica Santa Clara está ligada ao processo de migração reprodutiva em direção às corredeiras localizadas a montante do reservatório, entre as cidades de Carlos Chagas e Nanuque. Nesta região são encontrados, entre novembro e março, grandes cardumes de indivíduos em processo final de maturação gonadal ou após a reprodução, bem como grande volume de ovos e larvas destas espécies.

No caso das espécies marinhas, sua presença no rio Mucuri está provavelmente ligada ao seu uso como lar de alimentação já que sua desova não ocorre em água doce. No caso dos centropomídeos, a desova e desenvolvimento das formas iniciais ocorrem principalmente em regiões de mangue (TUCKER 
Tabela III. Abundância relativa de lambaris e de espécies migradoras nos ciclos de transposição em que foram transpostos mais de mil indivíduos.

\begin{tabular}{|c|c|c|c|c|c|c|c|c|}
\hline \multirow{2}{*}{ Dia } & \multirow{2}{*}{ Hora } & \multirow{2}{*}{ N Total } & \multicolumn{6}{|c|}{ Número relativo de indivíduos por espécie } \\
\hline & & & A. bimaculatus & A. intermedius & B. ferox & L. conir & L. steindachner & P. vimboides \\
\hline 06/jan & $12: 00$ & 2431 & - & 0,02 & 0,01 & 0,24 & 0,03 & 0,71 \\
\hline 08/jan & $12: 00$ & 2784 & - & - & 0,08 & 0,06 & 0,04 & 0,78 \\
\hline 09/jan & $16: 00$ & 1094 & - & - & 0,03 & 0,02 & 0,04 & 0,89 \\
\hline 17/jan & $14: 00$ & 1615 & - & - & 0,06 & 0,12 & 0,23 & 0,53 \\
\hline 17/jan & $16: 00$ & 1761 & - & - & 0,11 & 0,18 & 0,16 & 0,50 \\
\hline $21 /$ jan & $16: 00$ & 1149 & - & - & 0,03 & 0,05 & 0,02 & 0,79 \\
\hline 22/jan & $14: 00$ & 2361 & - & - & 0,01 & $<0,01$ & $<0,01$ & 0,96 \\
\hline $29 / \mathrm{fev}$ & $16: 00$ & 2614 & - & 0,99 & - & $<0,01$ & $<0,01$ & $<0,01$ \\
\hline $01 /$ mar & 08:00 & 3062 & - & 0,98 & - & $<0,01$ & $<0,01$ & $<0,01$ \\
\hline $02 / \mathrm{mar}$ & $14: 00$ & 3003 & - & 1,00 & - & - & - & - \\
\hline $02 / \mathrm{mar}$ & $16: 00$ & 1207 & $<0,01$ & 1,00 & - & - & - & - \\
\hline 03/mar & $18: 00$ & 1509 & 0,99 & - & - & $<0,01$ & - & - \\
\hline 03/mar & $22: 00$ & 2031 & 0,98 & - & - & $<0,01$ & - & - \\
\hline 03/mar & $24: 00$ & 1028 & 0,97 & - & - & - & - & - \\
\hline 04/mar & 08:00 & 1535 & - & 0,98 & - & $<0,01$ & $<0,01$ & $<0,01$ \\
\hline 04/mar & 10:00 & 1504 & - & 1,00 & - & - & $<0,01$ & - \\
\hline 04/mar & $12: 00$ & 5021 & $<0,01$ & 1,00 & - & $<0,01$ & $<0,01$ & $<0,01$ \\
\hline 05/mar & 08:00 & 2515 & - & 0,99 & - & $<0,01$ & $<0,01$ & $<0,01$ \\
\hline 05/mar & $14: 00$ & 2009 & - & 0,99 & - & $<0,01$ & - & $<0,01$ \\
\hline
\end{tabular}

Tabela IV. Abundância e captura por unidade de esforço em número por espécie, para os períodos diurno e noturno.

\begin{tabular}{|c|c|c|c|c|c|}
\hline \multirow{2}{*}{ Espécies } & \multicolumn{3}{|c|}{ Abundância (N) } & \multicolumn{2}{|c|}{ CPUE } \\
\hline & Dia & Noite & $8: 00 h$ * & Dia & Noite \\
\hline \multicolumn{6}{|l|}{ Espécies diurnas } \\
\hline Astyanax intermedius & 17313 & 0 & 7462 & 29,44 & 0,00 \\
\hline Brycon ferox & 1048 & 0 & 27 & 1,78 & 0,00 \\
\hline Centropomus paralellus & 275 & 2 & 73 & 0,47 & 0,04 \\
\hline Centropomus undecimalis & 450 & 2 & 89 & 0,76 & 0,04 \\
\hline Cichla monoculus & 22 & 0 & 5 & 0,04 & 0,00 \\
\hline Cyphocharax gilbert & 427 & 1 & 11 & 0,73 & 0,02 \\
\hline Geophagus brasiliensis & 40 & 0 & 2 & 0,07 & 0,00 \\
\hline Leporinus conirostris & 5040 & 2 & 783 & 8,57 & 0,04 \\
\hline Leporinus steindachneri & 4284 & 0 & 659 & 7,28 & 0,00 \\
\hline Mugil curema & 153 & 0 & 19 & 0,26 & 0,00 \\
\hline Oligosarcus acutirostris & 47 & 0 & 1 & 0,08 & 0,00 \\
\hline Prochilodus affinis & 22 & 0 & 2 & 0,04 & 0,00 \\
\hline Prochilodus vimboides & 21465 & 12 & 1535 & 36,50 & 0,25 \\
\hline \multicolumn{6}{|l|}{ Espécies noturnas } \\
\hline Astyanax bimaculatus & 1748 & 3902 & 44 & 2,97 & 81,29 \\
\hline Trachelyopterus striatulus & 34 & 27 & 10 & 0,06 & 0,56 \\
\hline Pogonopoma wertheimeri & 399 & 164 & 107 & 0,68 & 3,42 \\
\hline Trichomycterus alternatus & 10 & 2 & 3 & 0,02 & 0,04 \\
\hline
\end{tabular}


Tabela IV. Continuação.

\begin{tabular}{|c|c|c|c|c|c|}
\hline \multirow{2}{*}{ Espécies } & \multicolumn{3}{|c|}{ Abundância (N) } & \multicolumn{2}{|c|}{ CPUE } \\
\hline & Dia & Noite & $8: 00 h$ * & Dia & Noite \\
\hline \multicolumn{6}{|c|}{ Espécies com período não determinado } \\
\hline Achirus lineatus & 1 & 0 & 0 & $<0,01$ & 0,00 \\
\hline Awaus tajassica & 5 & 0 & 1 & 0,01 & 0,00 \\
\hline Brycon vermelha & 1 & 0 & 1 & $<0,01$ & 0,00 \\
\hline Charax latus & 0 & 0 & 1 & 0,00 & 0,00 \\
\hline Gymnotus carapo & 0 & 1 & 0 & 0,00 & 0,02 \\
\hline Hoplias malabaricus & 11 & 1 & 12 & 0,02 & 0,02 \\
\hline Hypostomus affinis & 1 & 0 & 2 & $<0,01$ & 0,00 \\
\hline Hypostomus luetkeni & 1 & 0 & 0 & $<0,01$ & 0,00 \\
\hline Leporinus copelandii & 7 & 0 & 31 & 0,01 & 0,00 \\
\hline Oreochromis niloticus & 5 & 0 & 0 & 0,01 & 0,00 \\
\hline Pachyurus adspersus & 2 & 2 & 0 & $<0,01$ & 0,04 \\
\hline Pimelodella lateristriga & 2 & 0 & 2 & $<0,01$ & 0,00 \\
\hline Pseudoauchenipterus affinis & 2 & 7 & 6 & $<0,01$ & 0,15 \\
\hline Rhamdia quelen & 8 & 1 & 1 & 0,01 & 0,02 \\
\hline Total geral & 52825 & 4126 & 10887 & 89,82 & 85,95 \\
\hline
\end{tabular}

*Transposições realizadas às 8:00 $\mathrm{h}$, quando não eram efetuadas transposições noturnas e os peixes capturados eram, então, resultado da atração desde as 18:00 h do dia anterior. Estes dados não foram computados nos cálculos da CPUE.

\& CAmpbell 1998), enquanto em Mugilidae a desova ocorre no mar, com uma fase estuarial obrigatória para os juvenis (BIzERRIL \& Costa 2002). Desta forma, a necessidade de transposição destas espécies deve ser avaliada, já sua reprodução ocorre nas áreas estuarinas e, para estas espécies, o retorno para as áreas de jusante através das estruturas da barragem é pouco provável (POMPEu et. al. 2004). Por outro lado, espécies marinhas possuíam, no período anterior à construção da barragem, importância comercial relevante para a pesca praticada pelas comunidade localizadas a montante. A facilidade de manipulação e escolha dos exemplares a serem transpostos pelo elevador possibilita que, no futuro, práticas de manejo específicas para estas espécies sejam adotadas, por exemplo, limitando o número de exemplares transpostos.

O comportamento migratório influência diretamente os padrões de utilização de mecanismos de transposição de peixes (Northсоте 1998). Padrões de movimentação ao longo do dia, embora descritos para algumas espécies Norte Americanas e Européias (LucAs \& BARAS 2001), não estão claros para a maior parte dos migradores neotropicais. Algumas espécies de siluriformes, grupo reconhecidamente de hábitos noturnos (BURGUESs 1989), parecem utilizar os mecanismos de transposição preferencialmente à noite. Characiformes, por sua vez, parecem apresentar padrão mais diverso, com picos de passagem pelos mecanismos variando bastante entre as espécies (OldAni \& Baigún 2002, Vono et al. 2004). No caso do elevador de Santa Clara, todas as espécies de Characiformes consideradas potenciais migradores apresentaram padrão coincidente, com picos de abundância entre as 10:00 e 16:00 h.

Analisando o padrão diário de utilização do elevador da Usina Hidrelétrica Santa Clara, outro interessante aspecto ob- servado diz respeito às diferenças observadas para as duas espécies de Astyanax transpostas. Enquanto A. intermedius utilizou o elevador principalmente durante o meio do dia, $A$. bimaculatus foi capturada predominantemente entre as 18:00 e 24:00 h.

Quando ocorrem interações agonísticas entre vertebrados utilizando o mesmo recurso alimentar ou o mesmo habitat, diferenças no período de atividade podem ser interpretadas como um mecanismo de redução da competição (CAROTHERs \& JAKSIÉ 1984). Avaliando a interação competitiva entre $A$. fasciatus e $A$. bimaculatus no reservatório de Americana, sudeste do Brasil, ArCifa et al. (1991) concluíram que estas duas espécies podem reduzir a competição no reservatório através da segregação de habitats, principalmente pelos adultos. No caso do rio Mucuri, é possível que diferenças nos horários de maior atividade estejam atuando como mecanismo redutor de competição entre as espécies deste gênero.

Os movimentos migratórios dos peixes constumam involver sua agregação em grandes concentrações (LuCAs \& BARAS 2001), situação bem documentada para a América do Sul (Bonetto 1963, Bonetto \& Pignalberi 1964, Godoy 1967, Bonetto et al. 1971, Baley 1973, Goulding \& Carvalho 1982, Paiva \& Bastos 1982, Petrere 1985, Barthem \& Goulding 1997). O grande número de peixes transpostos em alguns poucos ciclos, e a predominância de poucas espécies nestes mesmos ciclos foram resultado provavelmente do deslocamento das espécies migradoras e de lambaris em grandes cardumes.

Também chama a atenção o pequeno número de indivíduos transpostos da vermelha Brycon vermelha Lima \& Castro, 2000, espécie classificada como vulnerável na lista da fauna brasileira ameaçada de extinção (MAchado et. al. 2005). Este 


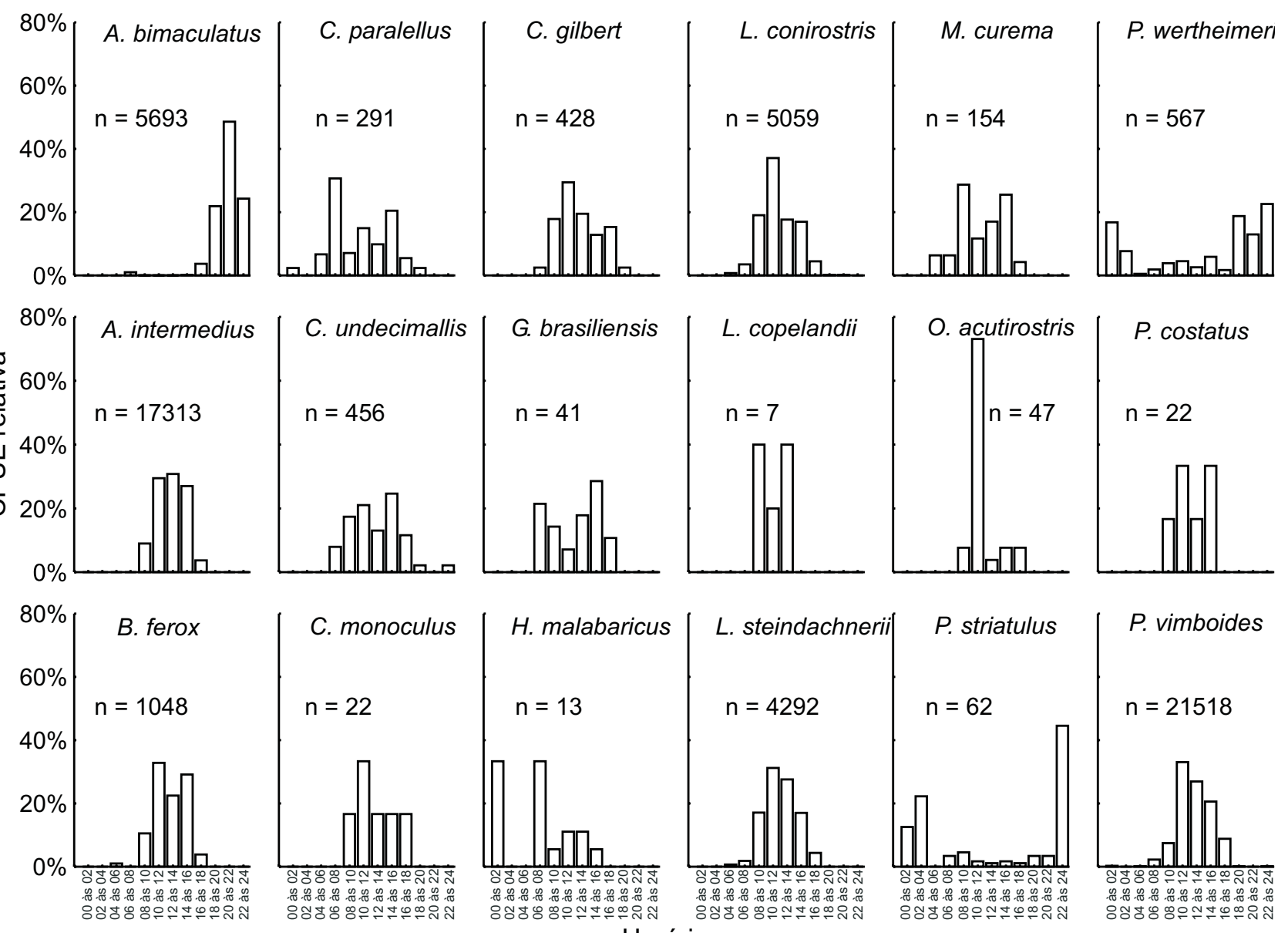

Horários

Figura 4. Distribuição das capturas por unidade de esforço em número ao longo do dia para as principais espécies de peixes transpostas pelo elevador da Usina Hidrelétrica Santa Clara. Não foram incluídos os dados das transposições realizadas às 8:00 h, quando não eram efetuadas transposições noturnas e os peixes capturados eram, então, resultado da atração desde as 18:00 h do dia anterior.

Tabela V. Relações estatísticas entre a abundância (CPUE) de diversos grupos de espécies transpostas e as vazões e precipitação no rio Mucuri. Variáveis transformadas para a escala logarítima; modelos significativos encontram-se em negrito.

\begin{tabular}{|c|c|c|c|c|c|c|c|c|}
\hline \multirow{2}{*}{ Espécies } & \multicolumn{2}{|c|}{ Vazão x CPUE } & \multicolumn{2}{|c|}{ Precipitação x CPUE } & \multicolumn{4}{|c|}{ Vazão e Precipitação x CPUE } \\
\hline & $\mathrm{p}$ & $r^{2}$ & $\mathrm{p}$ & $r^{2}$ & p modelo & p vazão & p precipitação & $r^{2}$ \\
\hline Migradores & 0,001 & 0,406 & 0,039 & 0,086 & 0,001 & 0,001 & 0,051 & 0,422 \\
\hline P. vimboides & 0,001 & 0,365 & 0,109 & 0,057 & 0,001 & 0,001 & 0,022 & 0,410 \\
\hline B. ferox & 0,003 & 0,199 & 0,649 & 0,009 & 0,021 & 0,007 & 0,205 & 0,285 \\
\hline L. conirostris & 0,001 & 0,138 & 0,309 & 0,023 & 0,036 & 0,018 & 0,338 & 0,140 \\
\hline L. steindachneri & 0,001 & 0,171 & 0,890 & 0,001 & 0,287 & 0,118 & 0,980 & 0,060 \\
\hline Marinhos & 0,001 & 0,463 & 0,049 & 0,077 & 0,001 & 0,001 & 0,081 & 0,549 \\
\hline Siluriformes & 0,400 & 0,010 & 0,004 & 0,206 & 0,009 & 0,240 & 0,006 & 0,237 \\
\hline Total & 0,001 & 0,415 & 0,071 & 0,065 & 0,001 & 0,001 & 0,140 & 0,456 \\
\hline
\end{tabular}

dado confirma observações anteriores de que a espécie tem distribuição preferencial nas porções mais altas da calha do rio Mucuri, em especial a montante da cidade de Carlos Chagas.
Os padrões temporais encontrados indicam que o estudo de uma regra operativa para o elevador em que sejam priorizados ciclos de transposição durante o dia e durante os períodos de 
maior vazão, apesar de valores de correlação relativamente baixos, poderá aumentar a eficiência do mecanismo, através do aumento do número de indivíduos de espécies migradoras transpostos. Quando comparado com o tipo de operação atual, de novembro a fevereiro e durante 24 horas, o funcionamento do mecanismo no período de maiores vazões e durante o dia poderia representar acréscimo de até 35\% no número de indivíduos de espécies migradoras transpostos, bem como uma economia em valor presente de até R\$960.000,00, considerandose taxas de juros anuais de $8 \%$ e vida útil do empreendimento de 30 anos (Pompeu \& Martinez 2005).

\section{AGRADECIMENTOS}

À Companhia Hidrelétrica Santa Clara (CESC), Construtora Queirós Galvão e Limiar Engenharia, que financiaram este trabalho em parceria com a UFMG, através do Centro de Estudos em Transposição de Peixes. Aos pescadores Ivanildo, Márcio, Menguinha, Gilberto, Ranieri, Ademir, Dedé e Isopor, que auxiliaram nos trabalhos de campo.

\section{REFERÊNCIAS BIBLIOGRÁFICAS}

Agostinho, A.A. 1994. Pesquisas, monitoramento e manejo da fauna aquática em empreendimentos hidrelétricos, p. 3859. In: COMASE (Ed.). Seminário sobre fauna aquática e o setor elétrico brasileiro: fundamentos, reuniões temáticas preparatórias: caderno 1 - Fundamentos. Rio de Janeiro, Eletrobrás, 61p.

Agostinho, A.A.; S.M. Thomaz; C.V. Minte-Vera \& K.O. Winemiller. 2000. Biodiversity in the high Paraná river floodplain, p. 89-118. In: B. GopAL; W.J. JunK \& J.A. DAvIS (Eds). Biodiversity in wetlands: assessment, function and conservation. New Delhi, School of Envirommental Sciences Jawaharlal Nehru University, vol. 1, 311p.

Arcifa, M.S.; T.G Northcote \& O. Froehlich. 1991. Interactive ecology of two cohabiting characin fishes (Astyanax fasciatus and Astyanax bimaculatus) in an eutrophic Brazilian reservoir. Journal of Tropical Ecology, New York, 7: 257-268.

Barthem, R. \& M. Goulding. 1997. The catfish connection: ecology, migration and conservation of Amazonian predators. New York, Columbia University Press, XVI+144p.

BAyley, P.B. 1973. Studies on the migratory characin, Prochilodus platensis Holmberg, 1889 (Pisces: Characoidei) in the R. Pylcomayo, South America. Journal of Fish Biology, Oxford, 5: 25-40.

Bittencourt, M.M. \& C. Cox-Fernandes. 1990. Peixes migradores sustentam pesca comercial. Ciência Hoje, Rio de Janeiro, 11 (64): 20-24.

Bizerril, C.R.S.F. \& P.R. Costa. 2002. Peixes marinhos do Estado do Rio de Janeiro. Rio de Janeiro, SEMADS/GTZ, 234p.

BonetTo, A.A. 1963. Investigaciones sobre migraciones de peces in los ríos de la cuenca del Plata. Ciencia e Investigación, Buenos Aires, 19 (1/2): 12-26.
BonetTo, A.A. \& C. Pignalberi. 1964. Nuevos aportes al conocimiento de las migraciones de los peces en los ríos mesopotámicos de la Republica Argentina. Comunicación Instituto Nacional de Limnologia, Santo Tomé, 1: 1-14.

Bonetto, A.A.; C. Pignalberi; E. Cordiviola de Yuan \& O. Oliveros. 1971. Informaciones complementarias sobre migraciones de peces en la cuenca del Plata. Physis, Buenos Aires 30 (81): 505-520.

BuRguess, W.E. 1989. An atlas of freshwater and marine catfishes. Neptune City, TFH, 785p.

Carothers, J.H. \& F.M. Jaksié. 1984. Time as a niche difference: the role of interference competition. Oikos, Lund, 42 (3): 403-406.

Cetec. 1983. Diagnóstico Ambiental do Estado de Minas Gerais. Belo Horizonte, Fundação Centro Tecnológico de Minas Gerais, 158p.

Clay, C.H. 1995. Design of Fishways and Other Fish Facilities. Boca Raton, CRC Press, 248p.

Esí́RITo SANTo. 1999. Regulamenta a implantação de mecanismos de transposição de peixes nos cursos de rios do estado do Espírito Santo. Decreto Normativo 4.489-N, de 15 de julho de 1999.

Fernandez, D.R.; A.A. Agostinho \& L.N. Binı. 2001. Influência dos fatores ambientais na ascensão de uma escada experimental de peixes da Hidrelétrica de Itaipu. In: Anais no XVI Seminário nacional de produção e transmissão de energia elétrica. Campinas, Eletrobrás. Disponível na World Wide Web em: http://www.xviiisnptee.com.br/acervo_ tecnico/memoria/xvi/11_Grupo_XI_Gia/gia-016.pdf [acesso em 05.IV.2006]

Godinho, A.L. 1993. E os peixes de Minas em 2010? Ciência Hoje, Rio de Janeiro, 16 (91): 44-49.

Godinho, H.P. \& A.L. GodinHo. 1994. Ecology and conservation of fish in southeastern Brazilian river basins submitted to hydroelectric impoundments. Acta Limnologica Brasiliensia, Rio de Janeiro, 5: 187-197.

Godoy, M.P. 1967. Dez anos de observações sobre periodicidade migratória de peixes do rio Mogi Guassu. Revista Brasileira de Biologia, São Carlos, 27: 1-12.

Goulding, M. 1979. Ecologia da pesca do rio Madeira. Manaus, CNPq/INPA, 172p.

Goulding, M. \& M.L. Carvalho. 1982. Life history and management of tambaqui (Colossoma macropomun, Characidae): a important Amazonian food fish. Revista Brasileira de Zoologia, São Paulo, 1: 107-133.

Lowe-McConnelL, R.L. 1999. Estudos ecológicos de comunidades de peixes tropicais. São Paulo, Edusp, 535p.

LuCAS, M.C. \& E. BARAS. 2001. Migration of freshwater fishes. Osney Mead, Blackwell Science, 420p.

Ludwig, J.A. \& J.F. REYNOLDs. 1988. Statistical Ecology: a primer on methods and computing. New York, John Wiley, 337p.

Machado, A.B.M.; C.S. Martins \& G.M. Drummond. 2005. Lista da fauna brasileira ameaçada de extinção: incluindo as 
espécies quase ameaçadas e deficientes em dados. Belo Horizonte, Fundação Biodiversitas, 160p.

Minas Gerais. 1997. Torna obrigatória a construção de escadas para peixes de piracema em barragem edificada no Estado. Lei $\mathbf{n}^{\circ}$ 12.488, de 09 de abril de 1997.

Northсоте, T.G. 1978. Migratory strategies in production in freshwater fishes, p. 326-359. In: S.D. GERKING (Ed.). Ecology of freshwater fish production. Oxford, Blakwell Scientific Publications, 520p.

Northсоте, T.G. 1998. Migratory behavior of fih and its significance to movement through riverine fish passage facilities, p. 3-18. In: M. JungwirTh; S. SCHMUTZ \& S. WEISS (Eds). Fish migration and fish bypasses. Oxford, Blakwell Scientific Publications, 438p.

Oldani, N.O. \& C.R.M. Baigun. 2002. Performance of a fishway system in a major South American dam on the Parana River (Argentina-Paraguay). River Research Applications, West Sussex, 18: 171-183.

Oldani, N; C. Baigún \& R. Delfino. 1998. Fishway performances in South American regulated rivers, p. 1129-1134. In: Wetlands Engineering and River Restoration Conference. Denver, Adams Mark, 1181p.

Paiva, M.P. \& S.A. BASTos. 1982. Marcação de peixes nas regiões do alto e médio São Francisco (Brasil). Ciência \& Cultura, Campinas, 34 (10):1362-1365.

PARÁ. 1995. Obriga a construção de escadas em barramentos. Lei $\mathbf{n}^{\circ} \mathbf{5 . 8 8 6}$ de 5 de abril de 1995 .

Petrere Jr., M. 1985. Migraciones de peces de agua dulce en America Latina: algunos comentarios. COPESCAL Documento Ocasional, Roma, 1: 1-17.

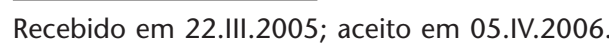

Pompeu, P.S. \& C.B. Martinez. 2005. Estabelecimento da regra operativa de um mecanismo de transposição de peixes do tipo elevador com caminhão-tanque. Revista Brasileira de Recursos Hídricos, Porto Alegre, 10 (4): 31-42.

Pompeu, P.S.; V.P. Ferreira; H.F. Silva \& C.B. Martinez. 2004. Evaluation of downstream fish passage through Santa Clara power plant dam, Minas Gerais, Brazil, p. 999-1003. In: Proceedings of Fifth International Symposium on Ecohydraulics, Aquatic Habitats: Analysis and Restoration. Madrid, IAHR, vol. 2, 1453p.

Quiros, R. 1988. Structures assisting the migrations of nonsalmonid fish: Latin America. FAO-COPESCAL Technical Document, Rome, (5): 1-50.

SALE, M.J. 1985. Aquatic ecosystem response to flow modification: an overview of the issues, p. 25-31. In: F.W. Olson (Ed.). Proceedings of the symposium on small hydropower and fisheries. Bethesda, American Fisheries Society, 497p.

São PAUlo. 1997. Dispõe sobre a construção de escadas para peixes em barragens edificadas em cursos d'água de domínio do estado. Lei $\mathbf{n}^{\circ} \mathbf{9 . 7 9 8}$ de 7 de outubro de 1997.

Tucker, JR., J.W. \& S.W. Campbell. 1988. Spawning season of common snook along the east central Florida coast. Florida Scientist, Tampa, 51(1): 1-6.

VAZzolER, A.E.A.M. 1996. Biologia da reprodução de peixes teleósteos: teoria e prática. Maringá, Eduem, 169p.

Vono, V.; P.M. Bizzotto; H.P. Godinho; A.L. Godinho \& B. Kynard. 2004. Fish passage at the Igarapava Fish Ladder, River Grande, Brazil, p. 1-5. In: Annals of the International Congress on the Biology of Fish Tropical Hotel Resort. Manaus, American Fisheries Society. 